\title{
Discrete stochastic models for traffic flow
}

\author{
M. Schreckenberg ${ }^{1}$, A. Schadschneider ${ }^{1}$, K. Nagel ${ }^{2}$, and N. Ito ${ }^{3, *}$ \\ ${ }^{1}$ Institut für Theoretische Physik, Universität zu Köln, D-50937 Köln, Germany, \\ schreck@thp.uni-koeln.de and as@thp.uni-koeln.de \\ 2 Zentrum für Paralleles Rechnen ZPR, Universität zu Köln, D-50923 Köln, Germany, \\ kai@zpr.uni-koeln.de \\ ${ }^{3}$ CISC JAERI, Tokai, Ibaraki 319-11, Japan, ito@catalyst.tokai.jaeri.go.jp
}

\begin{abstract}
We investigate a probabilistic cellular automaton model which has been introduced recently. This model describes single-lane traffic flow on a ring and generalizes the asymmetric exclusion process models. We study the equilibrium properties and calculate the so-called fundamental diagrams (flow vs. density) for parallel dynamics. This is done numerically by computer simulations of the model and by means of an improved mean-field approximation which takes into account short-range correlations. For cars with maximum velocity 1 the simplest non-trivial approximation gives the exact result. For higher velocities the analytical results, obtained by iterated application of the approximation scheme, are in excellent agreement with the numerical simulations.
\end{abstract}

\footnotetext{
*Present address: Dep. of Applied Physics, Faculty of Engineering, University of Tokyo, Tokyo 113, Japan
} 


\section{INTRODUCTION}

Recently, there has been considerable interest in the investigation of traffic flow using methods of statistical physics. Independently in [1] and [2] cellular automaton models [3] for the description of traffic flow have been proposed. Due to their computational simplicity, lattice gas automata [3] have already successfully been applied to other problems, e.g. the simulation of fluids [4] (for further applications, see the book of Wolfram [3]).

A similar class of discrete models — which may be interpretated as traffic models or as models for surface roughening — have also been used for the description of the so-called asymmetric exclusion process (driven diffusion) [5-13]. Here several exact solutions have been obtained for processes where the particles can move at most one lattice spacing per update step. A natural generalization would allow particles to move over larger distances. This is more realistic with regard to modelling traffic since one usually has a whole spectrum of allowed car velocities. These generalized exclusion models are thus more appropriate for comparison with 'experiments' (i.e. measurements on freeway traffic 14]).

In addition there have been a number of publications dealing with traffic flow in the framework of statistical mechanics, especially using cellular automata [1,2, 15-17]. In most of these works two-dimensional traffic has been studied which corresponds to modelling the complex situation of traffic in the street-network of a city. Again the cars were allowed to

move at most one lattice site per time step. It could be shown numerically [1] that relaxing this constraint, i.e. the cars can have integer velocities up to an upper speed limit larger than one, behaves qualitatively as well as quantitatively in good agreement with real traffic (with an appropriate upper speed limit). It will be shown in this paper that, from an analytical point of view, the situation changes drastically when turning from maximum velocity one to higher speed limits. In this case real long-range correlations occur even in the stationary state which is not true for models with maximum velocity one.

The exclusion models mentioned above may be classified according to the boundary conditions and dynamics used. There are two relevant types of boundary conditions: pe- 
riodic and open. Using periodic boundary conditions one considers a ring on which the cars/particles can move ('Indianapolis situation'). Open boundaries correspond to the socalled 'bottleneck situation' where one imposes certain input and output flows at the chain ends.

Basically one has to distinguish four types of dynamics. The dynamical variables may be updated one after the other in a certain order (sequential update), one after another in random order (random-sequential), in parallel for all sites of a given sublattice (sublattice update) or in parallel for all sites (parallel update). In the case of asymmetric update rules and sequential dynamics one has to distinguish at least two cases: update in or opposite to the direction of the traffic motion. Note that for open boundary conditions sequential and parallel dynamics are identical if the update direction is the same as the direction of traffic flow. For periodic boundary conditions, however, they are not identical. Here parallel dynamics correspond to sequential dynamics with a special site which closes the ring. This site memorizes a car even if it moved away one time-step before. This creates an obstacle in the ring giving rise to a lower flow through this site compared with sequential dynamics in the direction of the motion.

In the present paper we use periodic boundary conditions and parallel update. In this case not much is known exactly, since all of the previous works [5-13] used either randomsequential or sublattice update. The advantage of parallel update with respect to sublattice or sequential update is that all sites are equivalent which should be the case in a realistic model with translational invariance. On the other hand parallel update enhances the possible system sizes in numerical simulations, especially because parallel or vector computers can be used easily.

The paper is organized as follows: In Sect. 2 we introduce the model. In Sect. 3 the results of computer simulations are presented. We describe the different simulation techniques which have been used and compare their performance. In Sect. 4 we present a mean-field analysis of the model for arbitrary velocities $v_{\max }$. In Sect. 5 we introduce the so-called $n$-cluster approximation [18]. This improved mean-field method takes into account short- 
range correlations. In the case $v_{\max }=1$ we show that the 2 -cluster approximation already gives the exact result. For $v_{\max }=2$ we compare the results of the $n$-cluster approximation $(n=1, \ldots 6)$ with the computer simulations of Sect. 3 and find an excellent agreement. In App. A the exact solution for the case $v_{\max }=1$ is derived whereas in App. B the equivalence of this case to an Ising model with repulsive interactions is shown.

\section{THE MODEL}

In the following we study single-lane traffic on a ring of length $L$ with periodic boundary conditions. The model which has been introduced in [1] is defined as follows:

Each of the $L$ sites can either be empty or occupied by one vehicle with velocity $v=$ $0,1, \ldots, v_{\max }$. At each discrete time-step $t \rightarrow t+1$ an arbitary arrangement of $N$ cars is updated according to the following rules:

1) Acceleration: If the velocity $v$ of a vehicle is lower than $v_{\max }$ the speed is advanced by one $[v=v+1]$.

2) Slowing down (due to other cars): If the distance $d$ to the next car ahead is not larger than $v(d \leq v)$ the speed is reduced to $d-1[v=d-1]$.

3) Randomization: With probability $p$, the velocity of a vehicle (if greater than zero) is decreased by one $[v=v-1]$.

4) Car motion: Each vehicle is advanced $v$ sites.

These rules are applied to all cars in parallel (parallel update). The rules ensure that the total number $N$ of cars is conserved under the dynamics (which is not true in the bottlenecksituation). Note that even for parallel update the randomization yields non-deterministic behaviour. For random-sequential update the probability $p>0$ is not essential because it only rescales the time axis [1]. 
In the simplest case $v_{\max }=1$ the cars are allowed to move only one step during an update. For this situation several results are known [5-13]. Especially it can be shown that for random-sequential update the mean field Ansatz yields the exact equilibrium state [1.6] which is equivalent to the fact that for a fixed number of cars every arrangement of cars occurs with the same probability. Therefore it is quite natural to take the mean-field approach also as a starting point for the investigation of higher velocities $v_{\max }>1$ and parallel update.

Our main interest will be the calculation of the so-called fundamental diagram (flow $q$ vs. density $\rho=N / L$ ). As described in [四] these results can be compared directly with measurements of real traffic [14]. One expects a transition from laminar flow to start-stop waves with increasing car density. For $v_{\max }=1$ it is easy to see that the fundamental diagram is symmetric with respect to $\rho=1 / 2$ due to particle-hole symmetry. This is not true for realistic traffic where on finds a distinct asymmetry. i.e. the maximum of the flow is shifted to lower values of $\rho(\sim 0.2)$.

The rules given above take into account the basic features of real traffic. Firstly, they allow for a spectrum of velocities which seems to be necessary in order to break the particle hole symmetry of the model with only two velocities $v_{\max }=1$. The maximum velocity still enters the system as a free parameter but it can be argued [1] that most realistic are values around $v_{\max }=5$. Secondly, the acceleration of the cars is very smooth compared to the deceleration which can occur in only one time step. For simplicity we assume the same maximum velocity for all cars but this condition is not essential. Finally the randomization step is necessary to avoid purely deterministic dynamics and to take into account natural fluctuations of driving.

The order of the update rules given above is crucial. Changing it would also change the performance of the model. The randomization step could e.g. be placed between acceleration and deceleration (1-3-2-4) but then its influence is lowered drastically since every decelerating car does not feel any randomness. On the other hand one could also change the starting step. This will not influence the properties of the model but can simplify the 
calculations. If one begins with step 2 (as we will do for the $n$-cluster approximation) and proceeds $3-4-1$ then one has the advantage that no cars with velocity zero occur since all cars were just accelerated by one unit. Therefore the number of possible states of a site is reduced by one.

\section{THE SIMULATIONS}

An integral part of our research were computer simulations. Simulations allow to obtain quantitative insight into a model in relatively short time. In this way, they complement the analytical work: Simulations are first used to test a variety of models until one has some overview and the most useful is found, and high quality simulation data are used to confirm analytical results (see Figs. 6 and 7). Further on, simulations are used to go beyond the analytically treated cases: either for variations/extensions of the model, or for more complicated quantities and issues such as the life-time distribution of the simulated traffic jams [19] or the possibility of self-organized criticality [20]. Last, but definitely no least, this work are the first steps towards an ultrafast microscopic simulation model for large traffic networks. We already have results for 2-lane-traffic 21 23] and for real world network implementations including ramps, intersections, and junctions [21,24,25].

\section{A. Simulation technicalities}

Initially, we used a simple code on a workstation (and sometimes its replication on several nodes of a parallel computer) for getting an overview over the model's properties and for

estimating its relevance for real world traffic [四]. Later, we implemented vectorizing and/or parallelized versions in order to obtain faster simulation speeds.

Besides the advantage of getting high quality data in relatively short time, we have taken advantage of the simplicity of the model to implement it with different algorithms on many different supercomputers. This gives us intuition on how to implement more complex models [26], and to make predictions on how fast our simulations will be in comparison to 
other microscopic traffic simulation models [27]. In the cases of 2-lane-traffic and of the network implementation, our predictions were quite accurate.

For the practical coding, we considered three different approaches: site oriented, particle oriented, and an intermediate scheme. Site oriented directly implements the CA: A street is represented by a chain $\vec{v}$ of integers with values between -1 and $v_{\max }$, where -1 means that there is no particle at this site, whereas the other values denote a particle and its velocity. In contrast, particle oriented means that two lists $\left(x_{i}\right)_{i=1, \ldots, N}$ and $\left(v_{i}\right)_{i=1, \ldots, N}$ contain position $x_{i}$ and velocity $v_{i}$ of each particle $i(i=1, \ldots, N)$. This is similar to a molecular dynamics algorithm, except that particles are constrained to integer positions and velocities.

Obviously, the particle-oriented approach will always be faster than the site-oriented one for sufficiently low vehicle densities. The particle-oriented approach is more flexible, and since in single-lane simulations passing of vehicles is not possible, the particle lists are always ordered, making efficient codes for all kinds of computers easy to write. In addition, an extension to continuous position and velocity is straightforward [28].

On the other hand, for the site-oriented (cellular automaton) approach single-bit coding [29] is possible. This means that the model is formulated in logical variables, which may be stored bitwise into computer words. Logical operations on computer words treat all bits of the word simultaneously, giving a theoretical speedup of $b$, where $b$ is the number of bits per word (usually 32 or 64). However, the practical gain for traffic simulations on a workstation is much lower because the bit-oriented approach cannot take advantage of the fact that only a fraction of all sites is occupied by a particle. Nevertheless, we found that, on a workstation, the single-bit algorithm is faster for densities above 0.05 (for $v_{\max }=5$ ). In addition, the single-bit code runs very efficiently on a Thinking Machines CM-5 using dataparallel CM-Fortran and on a NEC-SX/3 traditional vector computer. The simulation data for the fundamental diagrams have been obtained this way.

Once passing of vehicles is allowed (multi-lane traffic), for the particle oriented approach efficient memory allocation for parallel and/or vector processors becomes more difficult, and single-bit coding for the site oriented approach becomes tiresome. These observations led 
to a third, intermediate approach. As in the site oriented approach, each site is in one of $\left(v_{\max }+2\right)$ states, but for the update only the relevant sites are considered. It turns out (see below) that on parallel but not vectorizing computers this algorithm is about as fast as the single-bit version.

In Table \&, we give an overview of the computational speeds on selected computers (see [27 for more details on most of these results). All values are valid for a vehicle density of $\rho=0.1$ and system size of $L=1333333$ sites, corresponding to 10000 single lane kilometers. MUPS corresponds to Mega-Updates Per Second, i.e., the number of sites updated per second divided by $10^{6}$. These values are useful in order to compare with other implementations of similar cellular automata or particle hopping systems. The other number is the extrapolated real time system size, i.e. the extrapolated system size (assuming linear speed-up) where the computation would be just as fast as reality.

The most noteworthy features of the table are emphasized in boldface:

- On vectorizing computers such as the SX-3 and the CM-5, the single-bit algorithm has a notable advantage over the other algorithms. On all other machines, the intermediate algorithm is never more than a factor 2.5 slower than the single-bit algorithm.

- Already on a relatively modest machine such as an Intel Paragon with 64 nodes, our real time limit is 280000 single lane kilometers. Since, e.g., the whole freeway network of Germany is about 60000 single lane kilometers long $(12000 \mathrm{~km} \times 2$ directions $\times 2.5$ lanes), the real use of this computational speed will be (i) real time applications, where the traffic forecast has to be computed before the situation arrives; and (ii) Monte Carlo simulations, where many runs are necessary.

- The by far fastest "realistic" traffic microsimulation world-wide to date is the PARAMICS microsimulation project [30]. Their real time limit is $\approx 20000 \mathrm{~km}$ on $16 \mathrm{k} \mathrm{CM}-200$, a machine which is in terms of peak performance slightly faster than a 128-nodeParagon. In other words, it seems that our not completely realistic car following logic buys us about an order of magnitude in performance. 


\section{B. Simulation results}

Figs. 1 and 2 show typical time evolutions of different versions of the model. Fig. 1 shows parallel update with $v_{\max }=1$ on the left, and random sequential update with $v_{\max }=5$ on the right, both at density $c=0.5$. Random sequential update for $v_{\max }=1$ looks the same as the left picture. Obviously, neither using parallel update for $v_{\max }=1$ nor taking a $v_{\max }>1$ for random sequential update does change the phenomenlogical behavior from the stochastic asymmetric exclusion process. For $v_{\max }=1, \rho=0.5$ corresponds to maximum flow, and in consequence, wave structures do not move in space [31]. For $v_{\max }>1$, the point of maximum throughput is shifted to lower densities, and in consequence, in the left plot the waves are moving backwards.

The situation is different when one combines parallel update and $v_{\max }>1$ (Fig. 2). Here, in the regime of maximum throughput (left plot) waves are only sparse, and they clearly move backwards. And even at higher densities (right plot), waves are much more distinct than in the random sequential case.

In short, one can divide the models between random sequential update with arbitrary maximum velocity on one hand, and parallel update with maximum velocity $v_{\max } \geq 2$ on the other hand. Parallel update with $v_{\max }=1$ phenomenologically is an intermediate case. Interestingly, it will turn out that this structure is reflected in the analytical calculations below: For random sequential update, the mean field solution is already exact. For parallel update and $v_{\max }=1$, the situation is only slightly different because already the first step beyond mean field is exact. The situation is completely different for higher velocities in connection with parallel update, where the analytical approximations only converge slowly towards the simulation result.

In addition, Fig. 2 allows an interesting comparison with fluid-dynamical models. Starting from ordered initial conditions, one clearly sees how instabilities develop and produce the start-stop-waves, very similar to results in [32]. Working out these connections is the topic of current research [25]. 
Fig. 3 (a) shows current vs. density curves for maximum velocities $v_{\max }$ between 1 and 5 , plus for a different fluctuation parameter $p=0.25$ at $v_{\max }=5$. One clearly sees that the maximum throughput increases with increasing $v_{\max }$, whereas the density of maximum throughput decreases. In reality, the density of maximum throughput lies between $\rho=$ 0.15 and $\rho=0.2$; it is given by the maximum speed of trucks which dominate the speed distribution for traffic near capacity [21]. For that reason, having a higher speed limit for passenger cars does not help throughput; in many cases, it actually makes things worse [33].

However, reducing the fluctuation parameter $p$ increases throughput enormously. This is mostly due to the better acceleration behavior in that case [34].

In general, one sees that by varying the parameters $v_{\max }$ and $p$, the fundamental diagram can easily be adapted to real traffic situations, although some of the underlying vehicle dynamics remain somewhat unrealistic: E.g. average acceleration from 0 to $100 \mathrm{~km} / \mathrm{h}$ takes place in 10 seconds. That fact that the fundamental diagram is nevertheless quite realistic is due to the fact that the first time steps fo the acceleration matter most [35]. And here, 4 seconds for an acceleration from 0 to $40 \mathrm{~km} / \mathrm{h}$ are far more realistic.

Another quantity of interest for traffic engineers are the velocity fluctuations

$$
\sigma\left(v_{l o c}\right):=\sqrt{\overline{\left(v_{l o c}-\overline{v_{l o c}}\right)^{2}}}
$$

where $v_{l o c}$ is the "local" velocity of vehicles crossing a fixed line. (The average of the local velocity is different from the usual ensemble average. For example, cars with velocity zero never enter the local average.) According to measurements and fluiddynamical arguments [33], these fluctuations are a good indicator of traffic near capacity. And indeed do we find for our model (Fig. $3(\mathrm{~b})$ ) that these fluctuations very abruptly increase near capacity.

\section{MEAN-FIELD THEORY}

The complete analytic solution of the traffic model is not possible except for the case of maximum velocity $v_{\max }=1$ (see Sect. 5.1 and Appendix A). Therefore some approximation 
is necessary when one tries to study this model analytically for $v_{\max }>1$. The following mean-field type theory will be the first step of such an analytical approach.

The calculation starts from the stochastic description of the traffic model. Instead of specifing the car position and their velocities, we analyze the probability distribution of each site at each time step. We denote the probability that there is no car at site $i(i=1,2,3$, $\cdots, L)$ at timestep $t$ by $d(i, t)$ and the probability that there is a car with velocity $\alpha(\alpha=0$, $\left.1,2, \cdots, v_{\max }\right)$ at site $i$ and timestep $t$ by $c_{\alpha}(i, t)$. The $c$ and $d$ distributions together take into account all possible states of the different sites. Therefore one has the normalization condition for all sites and all timesteps

$$
d(i, t)+c_{0}(i, t)+c_{1}(i, t)+c_{2}(i, t)+c_{3}(i, t)+\cdots c_{v_{\max }}(i, t)=1 .
$$

Denoting with $c(i, t)$ the total probability for site $i$ to be occupied at timestep $t$, i.e. $\sum_{j=0}^{v_{\max }} c_{j}(i, t)$, one simply has $d(i, t)+c(i, t)=1$.

According to the update rules in four stages (see Sect. 2) the time evolution of these probability distributions can be described by the following four sets of equations:

- Acceleration Stage

$$
\begin{aligned}
c_{0}\left(i, t_{1}\right) & =0 \\
c_{\alpha}\left(i, t_{1}\right) & =c_{\alpha-1}(i, t), \quad 0<\alpha<v_{\max } \\
c_{v_{\max }}\left(i, t_{1}\right) & =c_{v_{\max }}(i, t)+c_{v_{\max }-1}(i, t)
\end{aligned}
$$

- Deceleration Stage

$$
\begin{aligned}
c_{0}\left(i, t_{2}\right) & =c_{0}\left(i, t_{1}\right)+c\left(i+1, t_{1}\right) \sum_{\beta=1}^{v_{\max }} c_{\beta}\left(i, t_{1}\right) \\
c_{\alpha}\left(i, t_{2}\right) & =c\left(i+\alpha+1, t_{1}\right) \prod_{j=1}^{\alpha} d\left(i+j, t_{1}\right) \sum_{\beta=\alpha+1}^{v_{\max }} c_{\beta}\left(i, t_{1}\right) \\
& +c_{\alpha}\left(i, t_{1}\right) \prod_{j=1}^{\alpha} d\left(i+j, t_{1}\right), \quad 0<\alpha<v_{\max } \\
c_{v_{\max }}\left(i, t_{2}\right) & =\prod_{j=1}^{v_{\max }} d\left(i+j, t_{1}\right) c_{v_{\max }}\left(i, t_{1}\right)
\end{aligned}
$$


- Randomization Stage

$$
\begin{aligned}
c_{0}\left(i, t_{3}\right) & =c_{0}\left(i, t_{2}\right)+p c_{1}\left(i, t_{2}\right) \\
c_{\alpha}\left(i, t_{3}\right) & =q c_{\alpha}\left(i, t_{2}\right)+p c_{\alpha+1}\left(i, t_{2}\right), \quad 0<\alpha<v_{\max } \\
c_{v_{\max }}\left(i, t_{3}\right) & =q c_{v_{\max }}\left(i, t_{2}\right)
\end{aligned}
$$

- Motion Stage

$$
c_{\alpha}(i, t+1)=c_{\alpha}\left(i-\alpha, t_{3}\right), \quad 0 \leq \alpha \leq v_{\max }
$$

The time $t$ is assumed to take on only integer values. $t_{1}, t_{2}$ and $t_{3}$ denote the intermediate time steps between $t$ and $t+1$ (sometimes defined as $t+1 / 4, t+1 / 2$ and $t+3 / 4$, respectively). These time-evolution equations conserve for periodic boundary conditions the total number of cars at each stage. This formulation of the dynamics neglects spatial correlations completely since one assumes that all expectation values factorize into local terms.

The variables $c$ and $d$ are real valued between 0 and 1 . The stochastic description originates from the stochastic nature of the third randomization step. The other three steps are purely deterministic.

These time evolution equations are non-linear and further analysis of the full system has not been successful up to now. However, in the stationary state, i.e. in the limit $t \rightarrow \infty$, the $c$ and $d$ distributions become homogeneous in space (for periodic boundary conditions) and therefore, apart from the time dependence, also the site dependence can be omitted. Using this and combining the four update steps one gets the following set of $v_{\max }+1$ equations:

$$
\begin{aligned}
& c_{0}=(c+p d) c_{0}+(1+p d) c \sum_{\beta=1}^{v_{\max }} c_{\beta} \\
& c_{\alpha}=d^{\alpha}\left[q c_{\alpha-1}+(q c+p d) c_{\alpha}+(q+p d) c \sum_{\beta=\alpha+1}^{v_{\max }} c_{\beta}\right], \\
& 0<\alpha<v_{\max }-1 \\
& c_{v_{\max }-1}=d^{v_{\max }-1}\left[q c_{v_{\max }-2}+(q c+p d)\left(c_{v_{\max }-1}+c_{v_{\max }}\right)\right] \\
& c_{v_{\max }}=q d^{v_{\max }}\left[c_{v_{\max }-1}+c_{v_{\max }}\right]
\end{aligned}
$$


These equations essentially describe the motion of a single car with statistical (densitity dependent) 'obstacles'. A remarkable feature of the equations is that they are linear when one specifies the density $c=1-d$ of cars. Therefore (4.6) can be recast in matrix form as $\mathbf{A} \vec{c}=\vec{c}$. The matrix $\mathbf{A}$ can be read off from (4.6), $\vec{c}$ is the vector with elements $c_{\alpha}$, $\alpha=0, \ldots, v_{\max }$. For small $v_{\max }$ we can invert $\mathbf{A}$ to find the densities $c_{\alpha}$ explicitly. For large values of $v_{\max }$ it is more convenient to write down a recursion relation in order to obtain the steady state solution.

From the first equation in (4.6) one can determine $c_{0}$ directly without knowledge of the other $c_{\alpha}^{\prime} s$ to give

$$
c_{0}=c^{2} \frac{1+p d}{1-p d^{2}}
$$

Using this result and the second equation of (4.6) one can also write down the expression for $c_{1}$

$$
c_{1}=q c^{2} d \frac{1+d+p d^{2}}{\left(1-p d^{3}\right)\left(1-p d^{2}\right)} .
$$

For $\alpha$ larger than one a recursion relation can be derived calculating $c_{\alpha}-d c_{\alpha-1}$ using again the second equations of (4.6)

$$
c_{\alpha}=\frac{1+(q-p) d^{\alpha}}{1-p d^{\alpha+2}} d c_{\alpha-1}-\frac{q d^{\alpha}}{1-p d^{\alpha+2}} c_{\alpha-2}
$$

for $\alpha=2,3, \cdots, v_{\max }-2$. Therefore, starting with the expressions (4.7), (4.8) for $c_{0}$ and $c_{1}$, one can estimate $c_{2}, c_{3}, \cdots, c_{v_{\max }-2}$ recursively. These results do not depend on the actual value of $v_{\max }$ and thus are valid generally (provided $v_{\max } \geq 2$ ).

Finally, from the last two equations of (4.6) one gets

$$
\begin{aligned}
c_{v_{\max }-1} & =\frac{1-q d^{v_{\max }}}{1-d^{v_{\max }-1}(q+p d)} q d^{v_{\max }-1} c_{v_{\max }-2} \\
c_{v_{\max }} & =\frac{q d^{v_{\max }}}{1-q d^{v_{\max }}} c_{v_{\max }-1} .
\end{aligned}
$$

The $v_{\max }$ dependence only occurs in these two quantities. In Fig. 团 some of the densities are shown for large $v_{\max }$. The densities of the fast cars go to zero rapidly, since one expects an 
exponentially fast decay from the recursion relations, (4.9)-(4.11). The contributions from cars with high velocities therefore are neglegible. We will mainly be interested in the flow $f(c, p)$, defined by

$$
f(c, p)=\sum_{\alpha=1}^{v_{\max }} \alpha c_{\alpha}
$$

In the limit of $v_{\max } \rightarrow \infty$ it is possible to carry on the analysis using the iteration equations (4.7)-(4.9) and the generating function

$$
g(z)=\sum_{\alpha=0}^{\infty} z^{\alpha} c_{\alpha}
$$

As usual one simply has $g^{\prime}(1)=f(c, p)$. The equations (4.7)-(4.9) now can be combined to give one single equation for $g(z)$

$$
g(z)[1-d z]-g(d z) d^{2}(1-z)[p+q z]=c^{2}+p c^{2} d(1-z)
$$

Since $v_{\max }$ is infinite one does not have to worry about the upper boundary equations (4.10) and (4.11) for $c_{v_{\max }-1}$ and $c_{v_{\max }}$ whose contribution is neglegible. One should notice that the generating function $g$ occurs with two different $\operatorname{arguments}(z$ and $d z$ ) which makes it impossible to solve this (linear) equation for $g(z)$ explicitly.

After differentiation an expression for $g^{\prime}(1)$ is obtained

$$
g^{\prime}(1)=d(1-p c)-g(d) \frac{d^{2}}{c}
$$

The problem therefore is reduced to find an expression for $g(d)$. This can be found by successive application of equation (4.14) with $z=d^{n}, n=1,2,3, \ldots$. The final result is an asymptotic expression for $g^{\prime}(1)=f(c, p)$

$$
f(c, p)=q c d\left[1+\sum_{n=1}^{\infty} d^{2 n} \prod_{l=0}^{n-1}\left(p+q d^{l}\right)\right]
$$

In Fig. 5 the flow $f$ is shown as a function of the concentration $c$ of cars for various deceleration probabilities $p$. The slope at the origin $(c \sim 0)$ of the fundamental diagram is infinite whereas the slope for $c \sim 1$ is $-q$. This mean-field result yields, compared with the simulation data shown above, much too small values of the flow. This can easily be understood 
since the reduction to a single car problem ignores all spatial correlations of the cars. Cars with high velocities tend to be equidistant and can therefore maintain a high velocity with a larger probability than in the mean-field system where it is much more difficult to accelerate and stay at high velocities over a certain period.

Furthermore, even for $v_{\max }=1$, the mean-field solution does not coincide with the exact result shown below. Note that for random sequential update the mean-field solution is exact for $v_{\max }=1$.

\section{IMPROVED MEAN-FIELD THEORIES}

In order to improve the simple mean-field theory of the preceeding section we have to take into account correlations between neighbouring sites [18.

We divide the lattice into segments or clusters of length $n(n=1,2, \ldots)$ such that two neighbouring clusters have $n-1$ sites in common. The probability to find in equilibrium a cluster in state $\left(\sigma_{1}, \ldots, \sigma_{n}\right)$ will be denoted by $P_{n}\left(\sigma_{1}, \ldots, \sigma_{n}\right)$. Due to the translational invariance of equilibrium state of the system with periodic boundary conditions one has not to specify the actual location of the $n$-spin cluster. In order to simplify the calculations we apply, as mentioned above, the four update-rules in the order 2-3-4-1 instead of 1-23-4 [18. This has the advantage that after one update cycle one ends up with step 1 and therefore no car has velocity 0 . It follows that every site $j$ is in one of the $v_{\max }$ states $\sigma_{j}=0,1, \ldots, v_{\max }$ where now 0 denotes an empty site. This change in the ordering finally has to be taken into account in the calculation of the flow.

The equilibrium probabilities for an $n$-site cluster are then determined by

$$
\begin{aligned}
P_{n}\left(\sigma_{1}, \ldots, \sigma_{n}\right)=\sum_{\left\{\tau_{j}\right\}} W\left(\sigma_{1}, \ldots, \sigma_{n} \mid \tau_{-v_{\max }+1}, \ldots, \tau_{n+v_{\max }}\right) \times \\
\times P_{2 v_{\max }+n}\left(\tau_{-v_{\max }+1}, \ldots, \tau_{n+v_{\max }}\right),
\end{aligned}
$$

where the probability $P_{2 v_{\max }+n}\left(\tau_{-v_{\max }+1}, \ldots, \tau_{n+v_{\max }}\right)$ for clusters of length $2 v_{\max }+n$ has to be expressed through the $n$-cluster probabilities $P_{n}\left(\tau_{1}, \ldots, \tau_{n}\right)$. This enlargement of the clus- 
ter length occurs since all cars which can drive into or out of the cluster $\left(\sigma_{1}, \ldots, \sigma_{n}\right)$ within the next timestep contribute to the transition rates $W\left(\left\{\sigma_{j}\right\} \mid\left\{\tau_{j}\right\}\right)$. Thus we have to take into account not only the given cluster but also the $v_{\max }$ sites to its left (with state variables $\left.\tau_{-v_{\max }+1}, \ldots, \tau_{0}\right)$ and the $v_{\max }$ sites to its right (with state variables $\tau_{n+1}, \ldots, \tau_{n+v_{\max }}$ ). The decomposition of the $\left(2 v_{\max }+n\right)$-cluster can be carried out by introducing the conditional probabilities

$$
P\left(\tau_{i} \mid \underline{\tau_{i+1}, \ldots, \tau_{i+n-1}}\right)=\frac{P_{n}\left(\tau_{i}, \tau_{i+1}, \ldots, \tau_{i+n-1}\right)}{\sum_{\tau} P_{n}\left(\tau, \tau_{i+1}, \ldots, \tau_{i+n-1}\right)}
$$

at the left side and

$$
P\left(\underline{\tau_{i}, \ldots, \tau_{i+n-2}} \mid \tau_{i+n-1}\right)=\frac{P_{n}\left(\tau_{i}, \tau_{i+1}, \ldots, \tau_{i+n-1}\right)}{\sum_{\tau} P_{n}\left(\tau_{i}, \ldots, \tau_{i+n-2}, \tau\right)}
$$

at the right side. With this definition we rewrite $P_{2 v_{\max }+n}$ (in the $n$-cluster approximation) in the following form:

$$
\begin{aligned}
P_{2 v_{\max }+n}\left(\tau_{-v_{\max }+1}, \ldots, \tau_{n+v_{\max }}\right)= & \prod_{i=-v_{\max }+1}^{0} P\left(\tau_{i} \mid \underline{\tau_{i+1}, \ldots, \tau_{i+n-1}}\right) \times \\
& \times P_{n}\left(\tau_{1}, \ldots, \tau_{n}\right) \prod_{i=1}^{v_{\max }} P\left(\underline{\tau_{i+1}, \ldots, \tau_{i+n-1}} \mid \tau_{i+n}\right) .
\end{aligned}
$$

The transition probability $W\left(\sigma_{1}, \ldots, \sigma_{n} \mid \tau_{-v_{\max }+1}, \ldots, \tau_{n+v_{\max }}\right)$ depends on the probability $p$ and vanishes if the configuration $\left(\tau_{-v_{\max }+1}, \ldots, \tau_{n+v_{\max }}\right)$ cannot evolve in one timestep into $\left(\sigma_{1}, \ldots, \sigma_{n}\right)$ according to the rules 1$\left.)-4\right)$ of Sect. 2 . If $W$ is non-zero it is of the form $p^{n_{1}} q^{n_{2}}$ with integers $n_{1}, n_{2}$ describing how many cars have to be decelerated $\left(n_{1}\right)$ through the randomization step when total number of cars which can drive is $n_{1}+n_{2}$. With the approximation (5.4) it is possible to write down a closed system of equations for the $n$ cluster probabilities $P_{n}\left(\sigma_{1}, \ldots, \sigma_{n}\right)$. The number of the equations is given by $\left(v_{\max }+1\right)^{n}$, the total number of possible configurations of $n$ site variable with $v_{\max }+1$ possible states (without change of the order of the update steps one would have $\left(v_{\max }+2\right)^{n}$ equations).

\footnotetext{
${ }^{1}$ In practice some of these equations turn out to be trivial so that the relevant number is less than $\left(v_{\max }+1\right)^{n}$.
} 
Due to the exponential growth with respect to $n$ one is, especially for larger $v_{\max }$, restricted to only small cluster lengths $n$ (For the realistic value of $v_{\max }=5$ one has for the 2-cluster approximation already 36 equations!)

The above approximation converges for $n \rightarrow \infty$ to the exact solution for an infinite system (i.e. in the thermodynamic limit $L \rightarrow \infty$. This approximation scheme is well known in the literature. It is analogous to the $(n, n-1)$-cluster approximation of [36], the $n-1$ step Markovian approximation of [37] or the local structure theory of [38]. It goes back to the probability path method introduced by Kikuchi [39].

With the knowledge of the $n$-cluster probabilities $P_{n}\left(\sigma_{1}, \ldots, \sigma_{n}\right)$ it is then easy to calculate the fundamental diagram, i.e. the flow $f$ as a function of the density $c$ of cars. Since we have changed the order of the update steps one has to take this into account by performing the steps 2-3-4 at the end since the last step must be 4 (三 car motion). After that one simply can calculate the density $c_{\alpha}$ of cars which will drive $\alpha$ sites in the next timestep

$$
c_{\alpha}=\sum_{\sigma_{2}, \ldots, \sigma_{n}} P_{n}\left(\alpha, \sigma_{2}, \ldots, \sigma_{n}\right)
$$

and then one proceeds as in the mean-field approximation eqn. (4.12) and calculates $f=$ $\sum \alpha c_{\alpha}$

$$
\text { A. } v_{\max }=1
$$

In the case $v_{\max }=1$ the site variables take on only the values $\sigma=0,1$ where $\sigma=0$ means no car and $\sigma=1$ a car with velocity one. In the 2-cluster approximation ((5.1) with $n=2$ ) one has, according to the above arguments, a system of 4 equations. This can be be reduced to only one equation for $P(1,0)$ very easily with the help of the relations

$$
\begin{aligned}
& P(1,0)=P(0,1) \\
& P(0,0)=1-c-P(1,0), \\
& P(1,1)=c-P(1,0) .
\end{aligned}
$$


The first equation is due to the 'particle-hole' symmetry $P(1,0)=P(0,1)$ (in a closed ring one must have the same number of $(0,1)$ and $(1,0)$ pairs, therefore occuring with the same probability). The other two equations describe the conservation of cars in the system. The remaining equation for $P(1,0)$ reads

$$
q P^{2}(1,0)-P(1,0)+c(1-c)=0 .
$$

In the thermodynamic limit we therefore find [18]

$$
P(0,1)=P(1,0)=\frac{1-\sqrt{1-4 q c(1-c)}}{2 q} .
$$

Going to the 3- and higher-cluster approximations one finds that the solution remains the same indicating that this is the exact result. In App. A we indeed prove that the solution (5.8) is exact in the thermodynamic limit. For finite systems the prove is also valid but one has to take into account an additional correction (normalization) due to the constraint of a fixed number $N$ of cars.

The correct result, valid for any system size, is is given by

$$
\mathcal{P}(N, L)=\frac{1}{\mathcal{N}} \sum_{\{\sigma\}}^{\prime} \prod_{j=1}^{L} P\left(\sigma_{j}, \sigma_{j+1}\right)
$$

where $\mathcal{N}$ denotes the normalization constant and the sum $\sum^{\prime}$ runs over all configurations with a fixed number $N$ of cars (i.e. $\sum_{j=1}^{L} \sigma_{j}=N$ ).

In contrast to random-sequential dynamics parallel dynamics leads to an effective attraction between 'particles' and 'holes' (i.e. $P(0) P(1)=c(1-c) \leq P(0,1))$ and thus to a higher flow. In App. B the mapping of this model to an equivalent Ising-model with antiferromagnetic next-nearest-neighbour interactions and an nonvanishing external field is shown. Due to the antiferromagnetic interactions the system shows an effective attraction of cars over two lattice sites, thus taking into account the well known effect of car bunching or platooning 40 .

In order to calculate the flow one first has to perform the steps 2-3-4 yielding the probabilities $\tilde{P}(\tau)$ to find a site in the state $\tau=-1,0,1$ (where now an empty site is denoted by $\tau=-1$ ) after the fourth step of the updating procedure: 


$$
\tilde{P}(-1)=1-c, \quad \tilde{P}(0)=q P(1,1), \quad \tilde{P}(1)=q P(0,1)
$$

Therefore the fow is finally given by

$$
f(p, c)=q P(1,0)=\frac{1-\sqrt{1-4 q c(1-c)}}{2} .
$$

In Fig. 6 the exact result for the flow is shown (from the 2-cluster approximation) in comparison to numerical simulations and the mean-field approximation $(p=1 / 2)$. One can see that the numerical and analytical data are in excellent agreement. In the deterministic case $p=0$ the flow is a linear function of $c: f=(1-|1-2 c|) / 2$ [18.

The mean velocity per car $\bar{v}$ is then

$$
\bar{v}=\frac{1}{\rho} \sum_{\tau=0}^{v_{\max }} \tau \tilde{P}(\tau)=\tilde{P}(1) / \rho .
$$

In the deterministic case $p=1$ this simplifies to

$$
\bar{v}= \begin{cases}1 & \text { for } 0 \leq c \leq 1 / 2 \\ (1-c) / c & \text { for } 1 / 2 \leq c \leq 1\end{cases}
$$

which is the result of [2].

$$
\text { B. } v_{\max }=2
$$

The case $v_{\max }=2$ is qualitatively very different from the case $v_{\max }=1$. The flow diagram is no longer symmetric around $c=1 / 2$. The $n$-cluster approximation seems not to become exact for any finite $n$, i.e. in this case long-ranged correlations are important. We have calculated the fundamental diagram for $n=1, \ldots, 5$. As shown in Fig. 7 the approximation converges fast to the results obtained by simulations. The difference between $n=4$ and $n=5$ is less then $1 \%$.

The observation that the approximation does not become exact for small $n$ reflects the fact that the physics for $v_{\max } \geq 2$ is distinctly different from the case $v_{\max }=1$. As explained in Sec. 2 the regime looks qualitatively different both from $v_{\max }=1$ (arbitrary update) as well as from random sequential update (arbitrary $v_{\max }$ ). Moreover, in the case $v_{\max } \geq 2$ 
(parallel update), jams show characteristic branching behavior which is not observed for $v_{\max }=1[20]$.

\section{CONCLUSIONS}

We have introduced and investigated a statistical model capable to describe accurately real traffic. Through the introduction of higher velocities it was possible to produce the asymmetric fundamental diagrams and the characteristic start-stop waves observed in real traffic. Through simulation, different regimes depending on the type of update and the maximum velocity have been identified. Realistic for traffic is a model with parallel stochastic update and a suitable choice of the maximum velocity $v_{\max }=5$. Already for $v_{\max }=1$ the stationary state is more complicated than for random sequential update. An effective 'antiferromagnetic' interaction between cars favors equal spacing and in consequence higher throughput.

Taking into account two-point correlations the case $v_{\max }=1$ can be solved exactly. This is no longer true for higher maximum velocties where correlations become long ranged. Nevertheless, it could be shown that the $n$-cluster approximation converges fast to the simulation data.

\section{ACKNOWLEDGMENTS}

This work was performed within the SFB 341 Köln-Aachen-Jülich supported by the DFG. KN is supported by the "Graduiertenkolleg Köln/St. Augustin". The Center for Parallel Computing ZPR Köln, the German National Research Center KFA Jülich, the "Rechenzentrum" of the University of Cologne, and the "Institut für Angewandte Informatik" of the University Wuppertal provided computer time. 


\section{APPENDIX A: EXACT SOLUTION FOR $V_{M A X}=1$}

In this Appendix we show that the stationary state of the model with $v_{\max }=1$ is in fact given by equation (5.6) with an appropriate normalization constant $Z$. The complete set of evolution equations for parallel update reads:

$$
P_{t+1}(\{\boldsymbol{\sigma}\})=\sum_{\{\boldsymbol{\tau}\}} W(\{\boldsymbol{\sigma}\} \mid\{\boldsymbol{\tau}\}) \cdot P_{t}(\{\boldsymbol{\tau}\})
$$

where $P_{t}(\{\boldsymbol{\sigma}\})$ denotes the probability for state $\{\boldsymbol{\sigma}\}=\left\{\sigma_{1}, \ldots, \sigma_{L}\right\}$ at time $t$. The transition probability $W(\{\boldsymbol{\sigma}\} \mid\{\boldsymbol{\tau}\})$ to move in one timestep from state $\{\boldsymbol{\tau}\}$ to state $\{\boldsymbol{\sigma}\}$ factorizes into local terms:

$$
W(\{\boldsymbol{\sigma}\} \mid\{\boldsymbol{\tau}\})=\prod_{i=1}^{L} W\left(\sigma_{i}, \sigma_{i+1} \mid \tau_{i}, \tau_{i+1}\right) .
$$

The only non-vanishing transition probabilities are given by:

$$
\begin{aligned}
& W(0,1 \mid 1,0)=1-p \\
& W(1,0 \mid 1,0)=p \\
& W\left(1, \sigma^{\prime} \mid \sigma, 1\right)=1 \\
& W(\sigma, 0 \mid 0,0)=1 \\
& W(0, \sigma \mid 0,1)=1, \quad \sigma, \sigma^{\prime}=0,1
\end{aligned}
$$

whereas $W\left(\tau, \tau^{\prime} \mid \sigma, \sigma^{\prime}\right)=0$ in all other cases. (Note that in eqn. (5) of ref. [1] due to a misprint a factor $\left(1-\sigma_{i+1}\right) / 2$ is missing on the right-hand side.) We now make the Ansatz that the probability in the stationary state $P(\{\boldsymbol{\sigma}\})$ factorizes into local two-site terms $P_{\sigma_{i} \sigma_{i+1}}$ :

$$
P(\{\boldsymbol{\sigma}\})=\prod_{i=1}^{L} P_{\sigma_{i} \sigma_{i+1}}
$$

with periodic boundary condition $\sigma_{L+1}=\sigma_{1}$. We will define $n_{\sigma, \sigma^{\prime}}(\boldsymbol{\sigma})$ as the number of pairs of next neighbours $\left(\sigma, \sigma^{\prime}\right)$ in a particular state $\boldsymbol{\sigma}$. Due to the particle-hole symmetry of the system one has $n_{01}=n_{10}$ and the following simple relations for the system size $L$ and the (conserved) number of particles $N$ hold: 


$$
\begin{aligned}
& L=2 \cdot n_{01}+n_{11}+n_{00} \\
& N=n_{01}+n_{11} .
\end{aligned}
$$

In the stationary state equation (A.1) becomes time-independent and the states $\{\boldsymbol{\tau}\}$ in the summation on the right-hand side can be classified according to the number of particles $l$ which have to be moved in order to obtain the new state $\boldsymbol{\sigma}$ :

$$
\begin{aligned}
& \left(P_{01} P_{10}\right)^{n_{01}} P_{11}^{n_{11}} P_{00}^{L-2 n_{01}-n_{11}}= \\
& \sum_{l=0}^{n_{01}} \sum_{\Delta} g\left(n_{01}, l, \Delta\right)(1-p)^{l} p^{n_{01}-l-\Delta}\left(P_{01} P_{10}\right)^{n_{01}-\Delta} P_{11}^{n_{11}+\Delta} P_{00}^{L-2 n_{01}-n_{11}+\Delta} .
\end{aligned}
$$

The summation index $\Delta$ is defined as $\Delta=n_{11}(\boldsymbol{\tau})-n_{11}(\boldsymbol{\sigma})$. Therefore the range of the $\Delta$ summation depends on the particular state $\boldsymbol{\sigma}$. The function $g\left(n_{01}, l, \Delta\right)$ counts the number of possible states $\boldsymbol{\tau}$ leading to state $\boldsymbol{\sigma}$ given fixed values of $n_{01}, l$ and $\Delta$ and reflects a kind of degeneracy. Summing $g$ over $\Delta$ yields simply

$$
\sum_{\Delta} g\left(n_{01}, l, \Delta\right)=\left(\begin{array}{c}
n_{01} \\
l
\end{array}\right)
$$

Dividing eqn. (A.7) by the left-hand side one gets

$$
1=\sum_{l=0}^{n_{01}} \sum_{\Delta} g\left(n_{01}, l, \Delta\right)(1-p)^{l} p^{n_{01}-l}\left(\frac{P_{00} P_{11}}{p P_{01} P_{10}}\right)^{\Delta} .
$$

Setting the expression in the parenthesis on the right-hand side to 1, (A.9) reduces with the help of (A.8) to an identity. Therefore the condition for $P\left(\sigma, \sigma^{\prime}\right)$ reads

$$
P_{00} P_{11}=p P_{01} P_{10}
$$

Note that $P\left(\sigma, \sigma^{\prime}\right)$ is not normalized for a finite system and therefore a normalization constant $\mathcal{N}$ has to be taken into account.

\section{APPENDIX B: MAPPING TO AN EQUIVALENT ISING-MODEL}

Introducing Ising-variables $\sigma_{i}= \pm 1$ instead of the lattice-gas variables $\tau_{i}=0,1\left(\sigma_{i}=\right.$

$\left.2 \tau_{i}-1\right)$ one can look at the steady state as the equilibrium distribution $P(\boldsymbol{\sigma}) \sim e^{-\beta H(\boldsymbol{\sigma})}$ 
of an Ising model with Hamiltonian $H=-J \sum_{i} \sigma_{i} \sigma_{i+1}+h \sum_{i} \sigma_{i}$ In order to determine the coupling constant $J$ and the external field $h$ one interpretes the local probabilities $P_{\sigma, \sigma^{\prime}}$ as transfer matrices $P_{\sigma, \sigma^{\prime}}=\alpha e^{-J \sigma \sigma^{\prime}-h\left(\sigma+\sigma^{\prime}\right) / 2},(\beta \equiv 1)$. From eqn. (A.10) it follows directly that $e^{4 J}=p$ or $J=\log (p) / 4<0$. Therefore the corresponding Ising model has antiferromagnetic interactions. According to eqns. (A.5) and (A.6) one has in addition

$$
\begin{aligned}
& 1=2 \cdot P_{01}+P_{11}+P_{00} \\
& \rho=P_{01}+P_{11} .
\end{aligned}
$$

Dividing the two expressions on both sides of the two equations gives one eqn. without the constant $\alpha$ to determine the external field as

$$
e^{h}=\frac{\sqrt{1-4(1-p) \rho(1-\rho)}-(1-2 \rho)}{2 \sqrt{p}(1-\rho)} .
$$

Therefore the steady state corresponds to an Ising-model with antiferromagnetic (repulsive) interactions. Due to the conservation of the total number of particles (cars) one has to impose the constraint of a fixed magnetization to the Hamiltonian. The normalization is then simply the partition function calculated under this constraint. 


\section{REFERENCES}

[1] Nagel K and Schreckenberg M 1992 J. Phys. I (France) 2221

[2] Biham O, Middleton A A and Levine D 1992 Phys. Rev. A 46 R6124

[3] Wolfram S 1986 Theory and Applications of Cellular Automata (Singapore: World Scientific)

[4] Frisch U, Hasslacher B and Pomeau Y 1986 Phys. Rev. Lett. 561505

[5] Janowsky S A and Lebowitz J L 1992 Phys. Rev. A 45618

[6] Gwa L-H and Spohn H 1992 Phys. Rev. A 46844

[7] Derrida B, Domany E and Mukamel D 1992 J. Stat. Phys. 69667

[8] Derrida B and Evans M R 1993 J. Phys. I (France) 3311

[9] Derrida B, Evans M R, Hakim V and Pasquier V 1993 J. Phys. A: Math Gen. 26, 1493

[10] Kandel D, Domany E and Nienhuis B 1990 J. Phys. A: Math Gen. 23 L755

[11] Schütz G and Domany E 1993 J. Stat. Phys. 72277

[12] Schütz G 1993 Phys. Rev. E 474265

[13] Schütz G 1993 J. Stat. Phys. 71471

[14] Hall F L, Brian L A and Gunter M A 1986 Transp. Res. A 20197

[15] Cuesta J A, Martínez F C, Molera J M and Sánchez A 1993 Phys. Rev. E 484175

[16] Nagatani T 1993 J. Phys. Soc. Jpn. 621085

[17] Nagatani T 1993 Physica A 198108

[18] Schadschneider A and Schreckenberg M 1993 J. Phys. A: Math Gen. 26 L679

[19] Nagel K 1994 Int. Mod. Phys. C 5567 
[20] Nagel K and Paczuski M, Emergent Traffic Jams (in preparation)

[21] Rickert M 1994 Simulationen zweispurigen Autobahnverkehrs mit Zellularautomaten Master Thesis University of Cologne

[22] Latour A 1993 Simulation von Zellularautomaten-Modellen für Mehrspurverkehr Master Thesis University of Cologne

[23] Rickert M, Latour A , Nagel K, and Schreckenberg M (in preparation)

[24] Nagel K and Rasmussen S 1994, in: Brooks R, Maes P (eds.) Proceedings of Alife 4 MIT press Cambridge MA USA

[25] Nagel K 1994 Ph.D. thesis (in preparation)

[26] TRANSIMS - The TRansportation ANalysis and SIMulation System project, Los Alamos National Laboratory

[27] Nagel K and Schleicher A 1994 Parallel Comput. 20125

[28] Nagel K and Herrmann H J 1993 Physica A 199254

[29] Stauffer D 1991 J. Phys. A: Math Gen. 2491909

[30] Wylie B J N, McArthur D and Brown M D PARAMICS parallelisation schemes EPPCPARAMICS-CT.10

[31] Krug J 1991 in: Riste T and Sherrington D (eds.) Spontaneous formation of space-time structures and criticality Kluwer Academic Publishers Netherlands 37

[32] Kerner B S and Konhäuser P 1994 Phys. Rev. E 5054

[33] Zackor H, Kühne R and Balz W 1988 Untersuchungen des Verkehrsablaufs im Bereich der Leistungsfähigkeit und bei instabilem Fluß Forschung Straßenbau und Straßenverkehrstechnik 524, Bundesminister für Verkehr Bonn-Bad Godesberg

[34] Nagel K 1994 Fast low fidelity microsimulation of vehicle traffic on supercomputers 
Paper No. 940901 Transportation Research Board Meeting Jan. 1994 Washington D.C. USA

[35] Piper H P 1991 Internationales Verkehrswesen 43489

[36] ben-Avraham D and Köhler J 1992 Phys. Rev. B 458358

[37] Crisanti A, Paladin G and Vulpiani A 1993 Products of Random Matrices in Statistical Physics (Berlin: Springer)

[38] Gutowitz H A, Victor D and Knight B W 1987 Physica D 2818

[39] Kikuchi R 1966 Prog. theor. Phys., Suppl. 351

[40] May A D Traffic flow fundamentals Prentice Hall, Englewood Cliffs, NJ, 1990

[41] Ben-Naim E, Krapivsky P L, and Redner S Kinetics of clustering in traffic flows condmat preprint 1994

[42] Nagatani T Bunching of cars in asymmetric exclusion models for freeway traffic (submitted PRE) 


\section{TABLES}

\begin{tabular}{|c|c|c|c|c|}
\hline & s.bit (F77) & particle (F77) & intermed. (C) & netw. (C) \\
\hline Sparc10 & $\begin{array}{l}\text { 4.0 MUPS } \\
30000 \mathrm{~km}\end{array}$ & 3.4 MUPS & $\begin{array}{l}1.9 \text { MUPS } \\
14000 \mathrm{~km}\end{array}$ & $\begin{array}{l}1.2 \mathrm{MUPS} \\
8800 \mathrm{~km}\end{array}$ \\
\hline $\begin{array}{l}\text { PVM } \\
(5 \times \mathrm{Sp} 10)\end{array}$ & $\begin{array}{l}19.0 \text { MUPS } \\
140000 \mathrm{~km}\end{array}$ & & $\begin{array}{l}8.9 \text { MUPS } \\
65000 \mathrm{~km}\end{array}$ & \\
\hline $\begin{array}{l}\mathrm{SX}-3 / 11^{(1)} \\
1 \mathrm{CPN}\end{array}$ & $\begin{array}{l}533 \text { MUPS } \\
4000000 \mathrm{~km}\end{array}$ & & $\begin{array}{l}\mathbf{2 . 8} \text { MUPS } \\
21000 \mathrm{~km}\end{array}$ & \\
\hline $\begin{array}{l}\text { GCel-3 } \\
1024 \text { CPNs }\end{array}$ & $\begin{array}{l}102 \text { MUPS } \\
750000 \mathrm{~km}\end{array}$ & $\begin{array}{l}211 \text { MUPS } \\
1550000 \mathrm{~km}\end{array}$ & $\begin{array}{l}121 \mathrm{MUPS} \\
900000 \mathrm{~km}\end{array}$ & \\
\hline $\begin{array}{l}\text { iPSC } \\
32 \mathrm{CPNs}\end{array}$ & $\begin{array}{l}83 \text { MUPS } \\
630000 \mathrm{~km}\end{array}$ & & $\begin{array}{l}35 \text { MUPS } \\
260000 \mathrm{~km}\end{array}$ & \\
\hline $\begin{array}{l}\text { Paragon } \\
64 \mathrm{CPNs}\end{array}$ & & & & $290000 \mathrm{~km}$ \\
\hline $\begin{array}{l}\mathrm{CM}-5^{(1)} \\
32 \mathrm{CPNs}\end{array}$ & $\begin{array}{l}173 \text { MUPS } \\
1300300 \mathrm{~km}\end{array}$ & & $\begin{array}{l}30 \text { MUPS } \\
220000 \mathrm{~km}\end{array}$ & \\
\hline $\begin{array}{l}\text { CM-5 } 5^{(1)} \\
1024 \text { CPNs }\end{array}$ & & & & {$[>1.7$ e $6 \mathrm{~km}$} \\
\hline
\end{tabular}

(1) $\mathrm{CPN}(\mathrm{s})$ has/have vector units (SIMD instruction set)

(2) using data parallel Fortran (CMF)

(3) using message passing (CMMD) 
TABLE I. Computational speed of different algorithms and different machines. The machines are a SUN Sparc10 Workstation, 5 coupled Workstations Sparc10 under PVM, a NEC SX-3/11 traditional vectorcomputer, a Parsytec GCel-3 massively parallel computer with 1024 relatively slow T805-CPU's, an Intel iPSC/860 Hypercube with 32 nodes, and Intel Paragon XP-10/S with 64 nodes, and a Thinking Machines CM-5 with 32 nodes, each node containing one Sparc processor and 4 vector units. — "s.-bit" refers to the single-bit coded site-oriented algorithm, "particle" and "intermed." to the particle-oriented and the intermediate one, and "netw." refers to a network implementation of the freeway network of Germany. - All values are valid for a vehicle density of $c=0.1$ and system size of $L=1333333$ sites, corresponding to 10000 single lane kilometers. MUPS corresponds to Mega-Updates Per Second, i.e., the number of sites updated per second divided by $10^{6}$. The other number is the extrapolated real time system size, i.e. the extrapolated system size (assuming linear speed-up) where the computation would be just as fast as reality. Values in brackets [] are estimates. 


\section{FIGURES}

FIG. 1. Evolution of different automata rules from an ordered initial state of density $c=0.5$. Particles are moving to the right. Left: Same as (stochastic) asymmetric exclusion, except that the update is parallel. The same plot for exact asymmetric exclusion looks similar. Note that waves do not move in space. Right: Same as asymmetric exclusion, except that the maximum velocity is 5. Waves are now moving backwards, indicating a density above maximum flow. - Neither the parallel update nor the higher maximum velocity alone are sufficient to change the qualitative dynamics of the asymmetric exclusion model.

FIG. 2. Evolution of our traffic model (maximum velocity $v_{\max }=5$, parallel update) for density $c=0.3$ (left) and $c=0.1$ (right) from ordered initial conditions. Random sequential update with $v_{\max }=5$ at density $c=0.3$ (not shown) looks qualitatively similar to Fig. 1 left, whereas at density $c=0.1$ and random sequential update the waves are moving to the right (not shown). The density of maximum throughput is much lower for the parallel update, and instead of the waves switching from backward to forward motion at this point, they tend to vanish completely (cf. [20]). Moreover, the right picture illustrates the instability mechanism similar to the continuous model of 32].

FIG. 3. (a) Fundamental diagrams flow $j$ vs. density $c$ for maximum velocities $v_{\max }=1,2, \ldots$, 5 , and for a different fluctuation parameter $p=0.25$ instead of 0.5 at $v_{\max }=5$. (b) Fluctuations of local speed as a function of density $\left(v_{\max }=5\right.$ and $\left.p=0.5\right)$.

FIG. 4. Partial densities $c_{0}(c), c_{1}(c), \ldots, c_{5}(c)$ (i.e. for velocities 0 to 5 ) for mean field approximation. $p=0.5$

FIG. 5. Flow for $v_{\max }=\infty$ in mean field approximation. From bottom to top, the randomization parameter $p$ is $0.1,0.3,0.5,0.7$, and 0.9 .

FIG. 6. Convergence of the $n$-cluster approximations to the simulation result for the case $v_{\max }=1$ and $p=0.5$. Already the 2-cluster approximation is exact. 
FIG. 7. Convergence of the $n$-cluster approximations to the simulation result for $v_{\max }=2$ and $p=0.5$. Already the 5-cluster approximation gives a good fit of the simulation data. 